\title{
Polymer-particle mixtures: Depletion and packing effects
}

\author{
M. Doxastakis, Y-L. Chen, O. Guzmán, and J. J. de Pabloa) \\ Department of Chemical and Biological Engineering, University of Wisconsin, Madison, \\ Wisconsin 53706-1691
}

(Received 22 January 2004; accepted 20 February 2004)

\begin{abstract}
The structure of polymers in the vicinity of spherical colloids is investigated by Monte Carlo simulations and integral equation theory. Polymers are represented by a simple bead-spring model; only repulsive Lennard-Jones interactions are taken into account. Using advanced trial moves that alter chain connectivity, depletion and packing effects are analyzed as a function of chain length and density, both at the bond and the chain level. Chain ends segregate to the colloidal surface and polymer bonds orient parallel to it. In the dilute regime, the polymer chain length governs the range of depletion and has a negligible influence on monomer packing in dense polymer melts. Polymers adopt an ellipsoidal shape, with the larger axis parallel to the surface of the particle, as they approach larger colloids. The dimensions are perturbed within the range of the depletion layer. (C) 2004 American Institute of Physics. [DOI: 10.1063/1.1704634]
\end{abstract}

\section{INTRODUCTION}

Systems of small particles dispersed in dilute or dense polymer mixtures are of scientific and industrial interest. Applications are encountered in a large amount of everyday products, ranging from biological systems (dilute solutions) to polymer-particle nanocomposites, where a material of specifically desired mechanical properties may be designed.

Recent theoretical models and simulation techniques have significantly enriched our understanding of the polymer structure in the vicinity of a nanoparticle. ${ }^{1-8}$ At low polymer densities, monomers are depleted in the vicinity of the impenetrable particle surfaces, thereby leading to an effective attraction between particles in the solution. The range of interparticle attraction depends strongly on the polymer concentration and on its chain length; the latter determines the depletion layer thickness in the dilute regime. As the polymer concentration in the system reaches the semidilute regime, the depletion layer thickness no longer depends on chain length, but rather on the correlation length $\xi$ of the polymers. In the melt regime, monomer packing effects become significant and give rise to a repulsive barrier in the effective potential between two nanoparticles.

The development of well-characterized systems consisting of hard-spherelike nanoparticles has permitted the experimental characterization of entropic, polymer-induced depletion effects on the stability of polymer-particle suspensions. Experimental physical trends of the fluid-fluid phase boundaries are in agreement with theoretical results employing liquid state integral equation theory ${ }^{4,9,10}$ and results of simulations. ${ }^{11-17}$ Theory and simulation have provided valuable insights into the interactions between polymers and colloids. However, previous studies have focused on systems where the polymer concentration is dilute to semidilute, ${ }^{18-20}$ and where the particles are large relative to the polymer chains. In the limit where polymer chains are large enough to

${ }^{\text {a)} E l e c t r o n i c ~ m a i l: ~ d e p a b l o @ e n g r . w i s c . e d u ~}$ wrap around the particles (the so-called "protein or nanoparticle limit") a detailed description of the arrangement of macromolecules at the segmental length scale (rather than the chain length scale) is still lacking. Lattice-based models cannot accurately capture depletion and packing effects for these systems due to spatial restrictions imposed by the underlying lattice resolution. ${ }^{5,7,8}$ Unfortunately, studies of the interactions of nanoparticles in a "sea" of long polymer chains at a fully atomistic level of detail are limited significantly by computational power.

Vacatello ${ }^{21-23}$ has performed a series of continuum MC simulations of polymer melts filled with solid fillers. The MC algorithms employed in that work are only partially able to equilibrate the long-range structure of long polymer molecules, leaving issues related to polymer sizes unanswered. A thorough analysis on the structure of polymer-nanoparticle systems has recently been conducted by Picu, ${ }^{8}$ who performed lattice simulations of long chains. In that work polymer chains are found to adopt ellipsoidal shapes that do not deform but rather rotate with the larger semiaxis in the direction tangential to the spherical surface. The chain size decreases in the direction of its larger semiaxis only when the distance between two fillers decreases below two bulk radii of gyration. Although these findings are important on their own, it is of interest to explore whether they are confirmed in off-lattice models.

In the last few years, novel Monte Carlo techniques have been designed to overcome some of the computational limitations encountered in molecular dynamics simulations. ${ }^{24-26}$ Systems of long macromolecules (linear polyethylene chains of 6000 monomers) have been recently studied in atomistic detail by using advanced Monte Carlo techniques i.e., end-bridging ${ }^{27,28}$ with parallel domain decomposition. ${ }^{29,30}$ End-bridging has been shown capable of equilibrating a variety of polymeric melts by altering chain connectivity and consequently leading to a controlled polydispersity. ${ }^{27,28,31-34}$ The double rebridging move, which is an extension of connectivity altering moves, has the advantage of maintaining 
monodispersity in the systems studied.$^{35}$ Polyethylene chains have been recently simulated using a configurational bias double rebridging method that is easily applicable to a variety of polymeric systems. ${ }^{36}$

The present work examines the depletion layer thickness, polymer structure near a single nanoparticle, and the chain orientation and deformation due to the presence of particles with off-lattice canonical Monte Carlo simulations. Advanced Monte Carlo techniques such as configurational bias for inner chain segments and double rebridging between two neighboring chains are employed to efficiently sample configurations and determine the monomer and chain density profile relative to the impenetrable spherical surface. These profiles are then fitted to theoretical expressions, and the depletion layer thickness is examined as a function of chain length and density. The structure of the polymers and their sizes as they approach the filler are also analyzed.

\section{METHODOLOGY}

\section{A. Simulation}

Polymers are modelled as bead-spring chains with repulsive Lennard-Jones interactions, truncated and shifted at the minimum,

$$
\begin{aligned}
& U_{n b}(r)=4 \varepsilon\left[\left(\frac{\sigma}{r-R_{r}}\right)^{12}-\left(\frac{\sigma}{r-R_{r}}\right)^{6}\right]+\varepsilon, \\
& r-R_{r}<2^{1 / 6} \sigma \\
& U_{n b}(r)=0, \quad r-R_{r} \geqslant 2^{1 / 6} \sigma
\end{aligned}
$$

where $R_{r}=0$ for monomer-monomer interactions. The bonding energy of interaction between consecutive monomers in a polymer chain is of harmonic form,

$$
U_{b}(r)=\frac{1}{2} k_{h}\left(r-2^{1 / 6} \sigma\right)^{2}
$$

with the elastic constant $k_{h}=10^{4}$.

A single colloid is placed in each simulation system. Using Eq. (1) and a value of $R_{r}$, the interaction between the colloid and the monomers is taken into account from the surface of the particle as illustrated in Fig. 1. For separations closer than $R_{r}+0.5 \sigma$ increasingly stronger repulsion is used to prevent any overlaps between monomers and the colloid.

All systems are simulated at a temperature that makes the Barker-Henderson mean collision diameter, ${ }^{37}$

$$
d(\beta)=-\int_{0}^{\infty}\left(e^{-\beta u_{n b}(r)}-1\right) d r
$$

equal to $\sigma$ for the nonbonded potential. This special temperature is given by $1 / \beta=k T=1.50579 \varepsilon$. This facilitates comparison of our results to available findings for hard spheres with corresponding diameters $d_{m}=\sigma$ for the monomer and $d_{c}=\sigma+2 R_{r}$ for the colloid.

All systems studied in this work consist of 14400 monomers and one colloid. The number of chains in the systems is varied according to chain length so that the total number of chain segments is kept constant. Chains of $N=16,32$, and 120 beads are studied at a range of densities, starting from the dilute regime ( $\rho \sigma^{3}=0.025$, where $\rho$ is the number density of monomers) to the melt regime $\left(\rho \sigma^{3}=0.6\right)$. At the

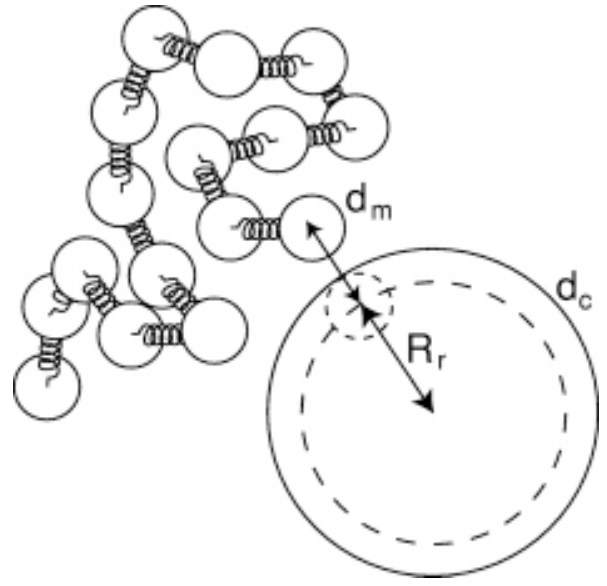

FIG. 1. Polymer-colloid interactions. Lennard-Jones interactions are taken from a distance $R_{r}$ from the center of the colloid using Eq. (1). The corresponding Barker-Henderson mean collision diameters are $d_{m}=\sigma$ for monomers and $d_{c}=\sigma+2 R_{r}$ for the colloid.

temperature studied, the good solvent scaling for the polymer size $\left(R_{g} \sim N^{0.6}\right)$ is recovered. The radius of the colloid $r_{c}$ is set to $2.45 \sigma$ so that the ratio $q=R_{g} / r_{c}$ of the chain radius of gyration at infinite dilution $\left(R_{g}\right)$ to the radius of the colloid $\left(r_{c}\right)$ are $q=1.00,1.59$, and 3.62 for $N=16,32$, and 120 , respectively. With this choice of nanoparticle size, the smallest polymer molecules studied have dimensions similar to the particle which can easily penetrate the volume spanned by longer chains.

A series of off-lattice canonical Monte Carlo (MC) simulations are conducted using a combination of trial moves to efficiently explore configuration space. Simple monomer and colloid displacements are used to alter the particle positions. Polymer chains diffuse through reptation moves, implemented within a biased scheme to increase their acceptance. The reptation move displaces a monomer from one chain end to the other chain end, placing it by selecting from a number of different attempted positions in a configurational biased fashion. Such a move is highly effective for the relaxation of small molecules $(N<50)$ but becomes progressively inefficient for longer chains. More sophisticated MC moves alter the chain conformation by rearranging a group of inner segments simultaneously. ${ }^{25}$ In particular, the double re-bridging move, a chain connectivity altering move ${ }^{36}$ that rearranges two neighboring chains by exchanging parts of them, is also used to improve sampling. The advantage of double rebridging vis-à-vis single end-bridging algorithms is that it preserves monodispersity, albeit at the expense of lower acceptance rates. Double rebridging moves permit rapid equilibration even in the large polymer systems considered in this work, thereby reducing the time needed to achieve sufficient sampling.

To demonstrate the efficiency of the MC method in equilibrating the systems under study, the orientational autocorrelation function $\langle\mathbf{u}(t) \cdot \mathbf{u}(0)\rangle$ of a unit vector directed along the chain end-to-end vector was evaluated as a function of number of simulation steps. Figure 2 shows the results for the three chain lengths considered here at the highest density $\left(\rho \sigma^{3}=0.6\right)$. The shortest chain systems exhibit 


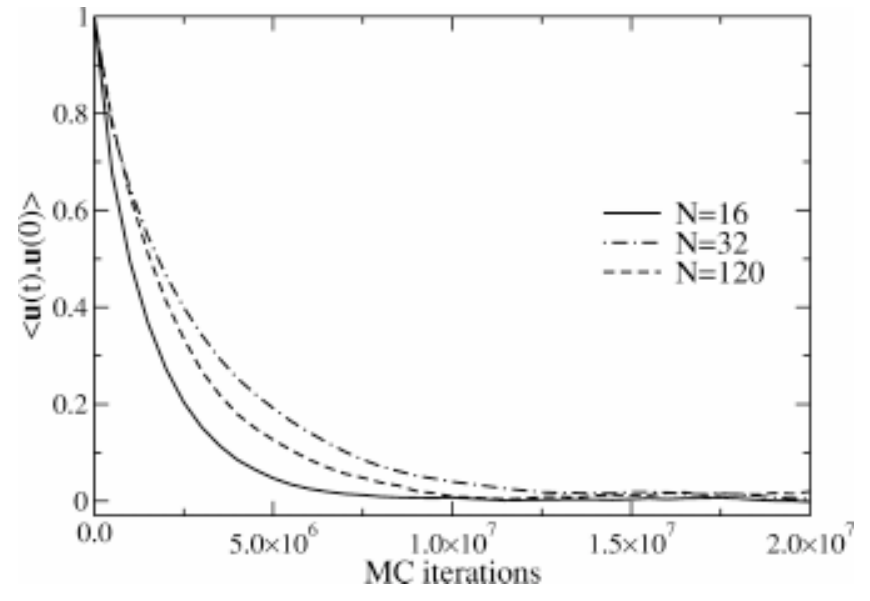

FIG. 2. Decay of the chain end-to-end vector orientational autocorrelation function $\langle\mathbf{u}(t) \cdot \mathbf{u}(0)\rangle$ for the three systems studied in this work at monomer number density $\rho \sigma^{3}=0.6$.

the fastest equilibration rate due to the configurational bias reptation move, which translates a significant part of the chain in a few steps. As we move to longer molecules, reptation becomes less efficient, rendering the relaxation of these systems slower $(N=32$ compared to $N=16)$. Intermolecular bridging moves such as double rebridging, however, equilibrate longer chain systems faster; ${ }^{27,28}$ this is illustrated in Fig. 2, where the calculated autocorrelation functions for $N=32$ and $N=120$ are compared. We note that the total length of our simulations varied between 250 million to 1 billion iterations, almost two orders of magnitude above the time needed for the end-to-end vector orientational autocorrelation function to drop to zero (around 10 million iterations, according to results shown in Fig. 2). Such a rapid decorrelation is indicative of good sampling and allows us to examine the structure of the systems with small statistical errors.

\section{B. Integral equation theory}

Recent comparisons between scattering experiments and liquid-state integral equation theory for dense particle suspensions with dilute polymer additives have shown that the theory accurately captures the collective particle structure factor. ${ }^{38,39}$ This approach has also been shown to correctly capture features of the fluid-fluid separation for a range of polymer radius of gyration to particle radius asymmetry ratios $(q=0.25-1.74) .{ }^{10,15}$ Prior integral equation studies of polymer-particle systems have also predicted repulsive barriers in the interparticle potential at high polymer concentrations. ${ }^{2}$ Here, the theory is employed to calculate the polymer-particle density pair correlation function and the depletion layer thickness for $q>1$ for subsequent comparison with simulation results.

More specifically, we use the polymer reference interaction site model (PRISM) ${ }^{40,41}$ For binary polymer-particle mixtures (the solvent is treated as a continuum that only modifies the polymer-polymer interactions), the intermolecular site-site pair correlation functions, $h_{i j}(r)=g_{i j}(r)$ -1 , intermolecular site-site direct correlations, $C_{i j}(r)$, and the single molecule structure factors, are related via the generalized Ornstein-Zernike matrix integral equation which in Fourier space are given by

$$
\hat{h}_{i j}(k)=\hat{\omega}_{i}(k)\left[\hat{C}_{i j}(k) \hat{\omega}_{j}(k)+\sum_{l} \hat{C}_{i l}(k) \rho_{l} \hat{h} s_{l j}(k)\right],
$$

where $k$ is the Fourier space wave vector and $\rho_{i}$ is the site number density of component $i$. For particles, the selfstructure factor is unity $\left(\omega_{c}(k)=1\right)$. The polymer chain is treated as a flexible pearl necklace of hard-sphere monomers with segment length $l=1.12 \sigma$ to be consistent with the simulation model. The polymer self structure factor $\omega_{p}(k)$ is obtained from the semiflexible chain model of Honnell et al. ${ }^{42}$ The persistence length of the chain is chosen to be $\xi_{p}=1.4$, which along with $l / \sigma=1.12$ accounts for chain flexibility and excluded volume through the average angle between any two connected segments, but does not account for excluded volume effects explicitly. Another approximation is that all sites on the chain are treated equally, thus neglecting effects due to chain ends.

To solve Eq. (4) the Percus-Yevick (PY) closure approximation ${ }^{43}$ is employed for the site-site direct correlation function with both the short-range Lennard-Jones (LJ) repulsive colloid used in the simulations and the pure hardcore interaction case (HS). This model has been shown to be in good agreement with simulation results for pure polymer melts, ${ }^{42}$

$$
\begin{aligned}
& C_{i j}(r)=\left(1-e^{\beta U_{i j}(r)}\right) g_{i j}(r), \\
& U_{i j}(r)=U_{n b}(r) \quad \text { PY-LJ, } \\
& U_{i j}(r)=U_{\mathrm{HS}}(r) \quad \text { PY-HS. }
\end{aligned}
$$

The hard-sphere potential is used for the monomermonomer direct pair correlation in both cases to allow the algorithm to converge. Picard's iteration method is used to solve this integral equation system.

\section{RESULTS}

\section{A. Local structure}

We first examine the structure of the systems in the vicinity of the nanoparticle, as revealed by the particlemonomer pair distribution function calculated from MC simulations. Figure 3 shows that for all the cases examined, the distance of closest approach is approximately equal to $0.5 \sigma$, i.e., the radius of a monomer. In the dilute regime $\left(\rho \sigma^{3}=0.025\right)$, chain segments are retracted or depleted near the particle due to a reduction of accessible chain conformations. An almost linear regime is observed from $0.5 \sigma$ (first monomer next to the colloid) to $\cong 1.6 \sigma$ (second monomer at $0.5 \sigma+1$ bond length) independently of chain length. The depletion of chain monomers continues up to a distance that is clearly different for the three chain lengths studied in this work, and the thickness of this depletion layer is roughly on the order of $R_{g}$.

At higher densities, the thickness of the layer decreases substantially and becomes almost independent of chainlength. Above the overlap density, polymers interpenetrate each other, thereby reducing the characteristic length of the 


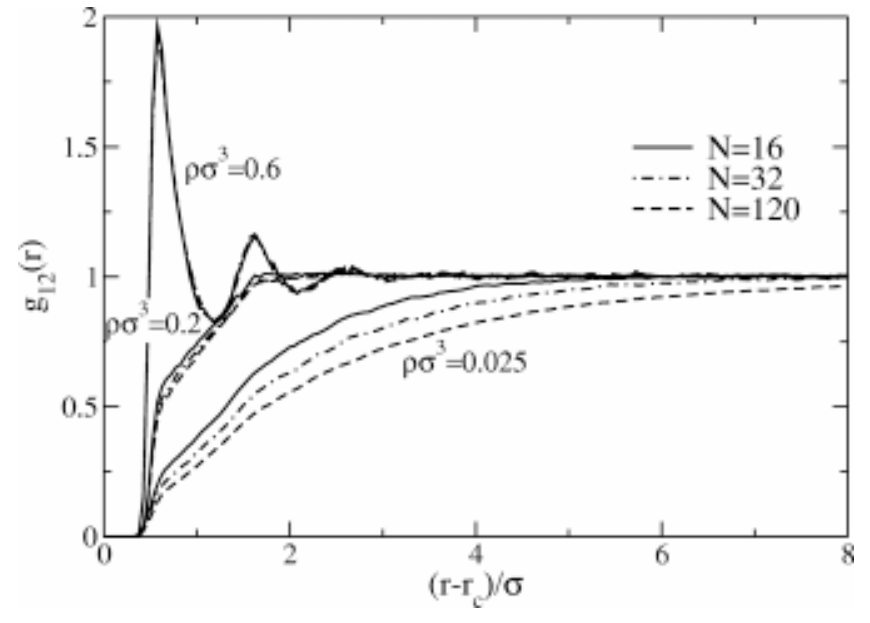

FIG. 3. Colloid-monomer pair distribution function for $\rho \sigma^{3}=0.025,0.2$, and 0.6 as calculated from the MC simulations for $N=16$ (solid line), 32 (dot-dashed line), and 120 (dashed line).

chain. ${ }^{44}$ At $\rho \sigma^{3}=0.2$, the polymer-particle correlation function $g_{12}(r)$ for different chain lengths are nearly indistinguishable, showing that in the semidilute regime the relevant length scale is indeed the density dependent correlation length $\xi(\rho)$. In the melt regime, monomer packing effects become increasingly important as monomers form layers around the nanoparticle; this is illustrated by the oscillations in $g_{12}(r)$. The first peak is located at contact; subsequent peaks are at integer multiples of bond length. The intensity of the peaks decreases as the chain length increases, but this effect is extremely weak and not distinguishable in the figure. These packing effects have already been observed in various continuum simulations of polymers close to impenetrable surfaces, but they are absent in lattice-model studies.

PRISM predictions for $g_{12}(r)$ are compared to results of MC simulations for $N=120$ in Fig. 4. Results are shown for both the PY closure with the interaction potentials $U_{i j}(r)$ $=U_{\mathrm{HS}}(r)$ (PY-HS) and $U_{i j}(r)=U_{n b}(r)$ (PY-LJ). In the dilute regime, the theory predictions are in excellent agreement

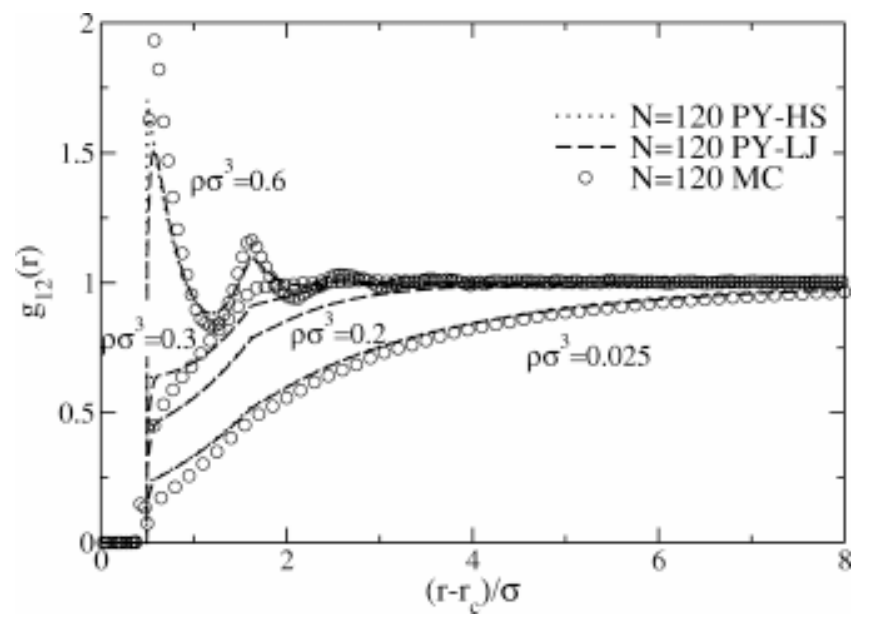

FIG. 4. Colloid-monomer pair distribution function for $\rho \sigma^{3}=0.025,0.2$, and 0.6 as calculated from MC simulations (open circles), and $\rho \sigma^{3}$ $=0.025,0.2,0.3$, and 0.6 (dashed and dotted lines) as calculated from integral equation theory for $N=120$. with the simulation results over a significant range. However, both PY-HS and PY-LJ overestimate $g_{12}(r)$ near polymerparticle contact. This observation agrees with prior PRISM studies that attribute this to the local nature of the PY closure, ${ }^{45}$ which cannot account for nonlocal correlations between the particle and monomer sites not in contact with the particle. A modified PY closure has been developed recently for polymer coil-particle systems to correct for this nonlocal effect, ${ }^{45,46}$ but the development of this new closure is beyond the scope of this study.

As the system moves into the semidilute regime, the theory predictions capture the qualitative shape of $g_{12}(r)$ but underestimates its value and the range of the depletion layer. This may be due to several differences between the theoretical model and the simulation model. In the theory, the polymer stiffness does not change when a polymer is near a particle or other polymers. The semiflexible chain model also allows nonphysical overlapping of adjacent monomer sites. These factors lead to lower polymer-particle correlation predictions and weaker interpolymer repulsions. As shown in the figure, PRISM predictions for $\rho \sigma^{3}=0.3$ are in near quantitative agreement with $\mathrm{MC}$ results for $\rho \sigma^{3}=0.2$. In the dense regime the theory again underestimates $g_{12}(r)$ near contact, but is in quantitative agreement with the simulation results over a significant range beyond contact. This may be attributed to the fact that segments near a surface cannot be as flexible as if they were far away, and the nonphysical monomer overlapping would reduce the polymer-particle correlation near contact. Theory predictions of $g_{12}(r)$ near contact with the Lennard-Jones potential are lower than the predictions with the hard core potential and the simulations, which indicates that at high densities, hard-core contributions to the polymer-particle correlation are dominant due to monomer packing. These quantitative differences between theory and simulations would also affect the determination of the depletion layer thickness.

The depletion layer thickness may be defined with a literal depletion zone of thickness $\delta$ where there are no monomers around a sphere of radius $r_{c} \cdot{ }^{47}$ Calculating the number of polymers depleted in this volume leads to an expression for the excluded volume of the form

$$
\frac{4 \pi}{3}\left(\left(r_{c}+\delta\right)^{3}-r_{c}^{3}\right)=4 \pi \int_{r_{c}}^{\infty} r^{2}\left(1-g_{12}(r)\right) d r .
$$

The monomer density profile in the vicinity of the particle can be approximated by the mean field equation, ${ }^{48}$

$$
g_{12}(z)=\left(\frac{z / r_{c}+\tanh (z / \delta)}{z / r_{c}+1}\right)^{2},
$$

where $z=r-r_{c}$. Although more accurate equations can be found in the literature, this form is simple and can be used to fit the simulation results in the dilute and semidilute regime. In Fig. 5, the calculated $g_{12}(r)$ functions from simulations (open circles) are fitted with the above equation for $N=16$ and $\rho \sigma^{3}=0.025,0.05,0.1$, and 0.2 to find $\delta$. The mean-field equation provides an excellent description for the three lower densities, while for $\rho \sigma^{3}=0.2$ it is clear that monomer packing has started to develop, leading to substantial deviations from the theory for polymer coils. For longer chain lengths, 


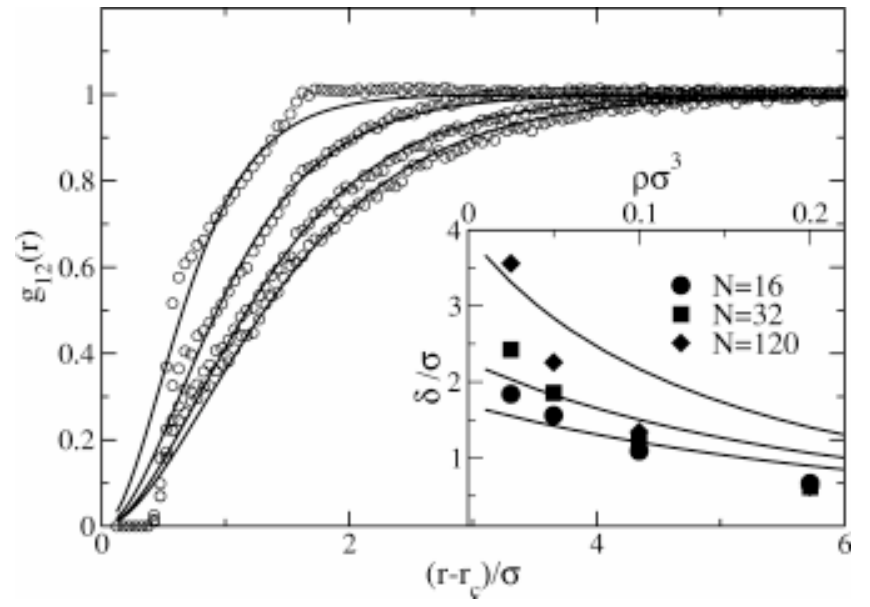

FIG. 5. Colloid-monomer pair distribution function for $\rho \sigma^{3}=0.025,0.05$, 0.1 , and 0.2 (open circles) as calculated from the MC simulations for $N$ $=16$. Thick lines are fits to the data using the mean field description provided by Eq. (7). The inset presents the depletion thickness $\delta$ extracted from the fits, as a function of chain length and density (filled symbols). Lines in the inset are predictions from PRISM.

simulation results also follow Eq. (7), although the quality of the fit decreases. The above procedure also allows us to estimate the thickness of the depletion layer as a function of chain length and density. Figure 5 compares the calculated values of $\delta$ from the MC simulations and from PRISM. In the dilute polymer regime, the thickness of the depletion layer increases with chain length (interaction range) as already stated. As polymer density increases, $\delta$ decreases down to around one bond length at $\rho \sigma^{3}=0.2$ for all chain lengths.

The pair distribution function for the colloid and polymer chain ends exhibits a similar profile to that of the segment density profile shown in Fig. 3. At low densities, chain ends are depleted in the vicinity of the spherical particle, while at high densities they are packed close to its surface. It is interesting to examine the magnitude of this effect for chain ends relative to any other segment. ${ }^{7}$ The fraction $f_{e}(r)$ of chain-ends density to monomer density is calculated and normalized to the bulk value to observe whether end monomers prefer to be close to the impenetrable surface more than the inner chain segments. The results are shown in Fig. 6 for the $N=16$ chain at the lowest and highest density studied as a function of distance from the filler's surface. For all the cases studied here, chain ends always prefer to be in the vicinity of the spherical particle relative to an inner monomer. At $\rho \sigma^{3}=0.025$, an end monomer is more than twice as likely to be in contact with the particle than an inner monomer. At high densities, where monomer packing is significant, the effect is less pronounced. The range of this preferential behavior is extended for a distance of one bond length, from $r-r_{c}=0.5 \sigma$ up to $r-r_{c} \cong 1.6 \sigma$. Figure 6 inset shows the end-to-inner monomer ratio at monomer-particle contact $\left(r=r_{c}+0.5 \sigma\right)$ for all the simulations performed. It is evident that as the bulk chain end concentration decreases by moving to longer chains, this preferential packing effect becomes more pronounced, as also observed by lattice simulations. ${ }^{8}$ The preference of chain ends near particles is not included in PRISM, which treat all sites on the chain equivalently, but its

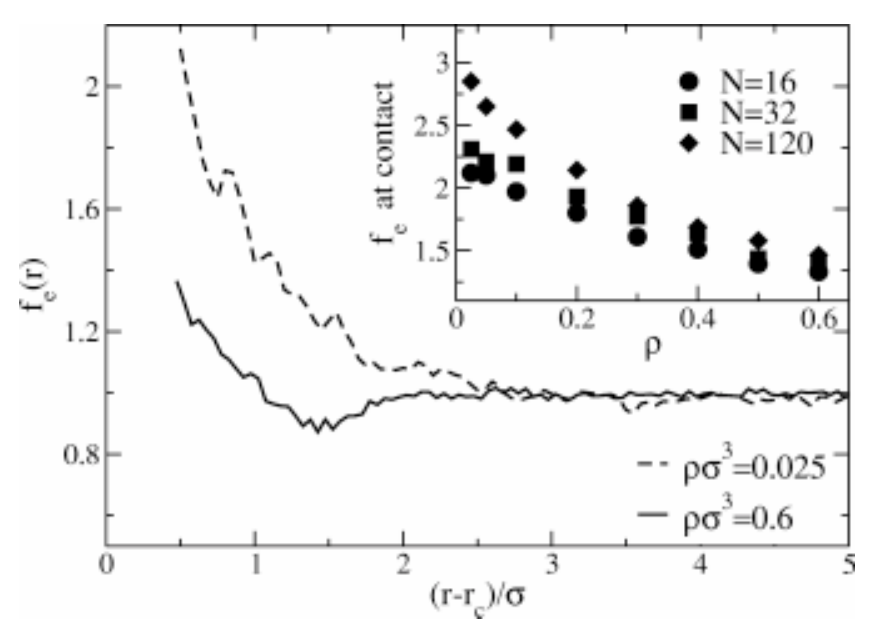

FIG. 6. Fraction of chain-ends to monomers normalized to the bulk value, as a function of distance from the colloid surface. The lines are results from the lowest (dashed line) and the highest density (continuous line) studied for $N=16$. In the inset, the value at contact $\left(r=r_{c}+0.5 \sigma\right)$ is plotted as a function of chain length and density.

contribution to the differences between PRISM and simulations is small, especially for long polymer chains. However, this effect may be significant for polyelectrolytes or polymers with functionalized end groups.

The orientation of the polymer segments next to the particle surface is examined by calculating the second Legendre polynomial $P_{2}=\frac{1}{2}\left(3 \cos ^{2} \theta-1\right)$ of the angle $\theta$ formed by the vector connecting the centers of the particle to monomer $i$ and the bond vector connecting monomer $i$ and the next monomer in the same chain, monomer $i+1$. The segment orientation parameter is defined by taking the average of $P_{2}$ for all the segments in the vicinity of the nanoparticle. A value of $\left\langle P_{2}\right\rangle=0$ indicates a random orientation of the segments, and a value of $\left\langle P_{2}\right\rangle=-0.5$ indicates perfect tangential alignment of all segments near the particle with the particle surface. Figure 7 shows the orientation of segments as

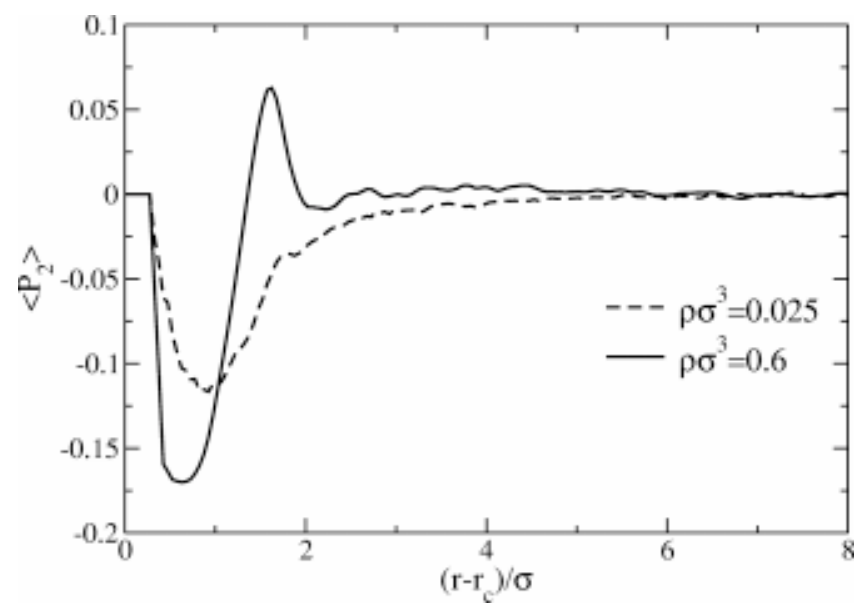

FIG. 7. Mean second Legendre polynomial $P_{2}$ between the vector connecting the center of the particle and a monomer $i$ in a polymer chain and the bond vector of monomer $i$ with the next monomer in the same chain, $i+1 . P_{2}$ is calculated as a function of distance of monomer $i$ from the center of the colloid. Data shown in the figure are from the MC simulations with $N=120$ at the lowest and highest densities studied in this work. 


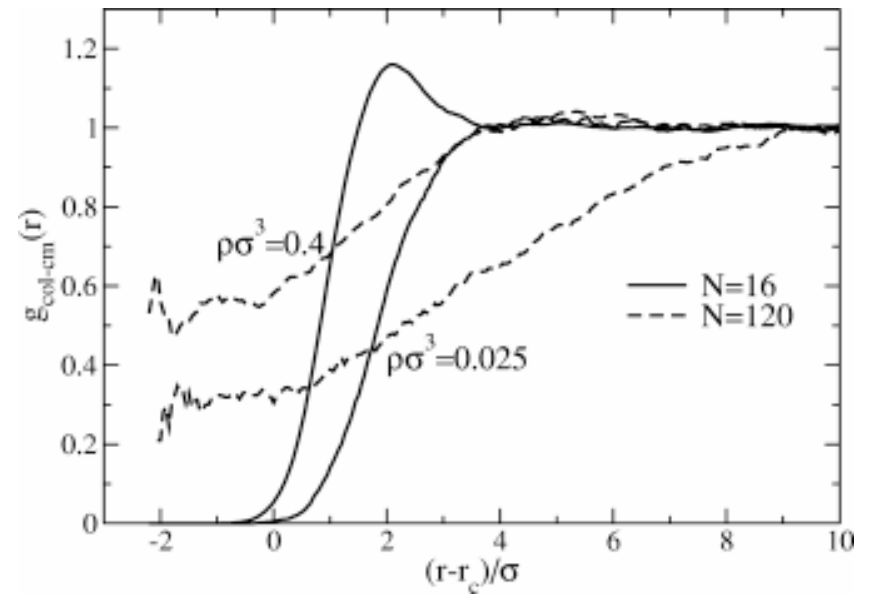

FIG. 8. Colloid-chain center of mass pair distribution functions for $N=16$ and $N=120$ at $\rho \sigma^{3}=0.025$ and $\rho \sigma^{3}=0.4$.

they approach the spherical particle. At all densities studied, the closest segments to the particle align parallel to the surface, as shown by the negative values of $\left\langle P_{2}\right\rangle$ at approximately $0.5 \sigma$. Very close to the surface, an upturn is observed due to restrictions of orientations of the bond vector that contribute to negative values. The preferential orientation is more pronounced at high densities where a positive peak appears for the second layer of bonds. Similar shapes have been observed for this function in off-lattice simulations of polymer melts between plates. ${ }^{49}$

\section{B. Chain structure}

At the chain size length scale, the structure of the systems can be examined by particle-chain-center-of-mass pair distribution functions, as shown in Fig. 8 for $N=16$ and $N$ $=120$. For the 16 -bead chains $\left(R_{g} \cong 2.45 \sigma, q=1\right)$, the polymer and particle centers of mass are rarely located at a distance below $r_{c}$ and there is little interpenetration. For $N$ $=120\left(R_{g} \cong 8.87 \sigma\right)$, Fig. 8 clearly shows that the population of centers of mass of the chains is nonzero for $r<r_{c}$, even at the most dilute case considered here, indicating that the chain can wrap around the particle and have a center of mass located "inside" the particle. The pair distribution function for long chains seems to follow an almost linear behavior with two separate regimes. One for distances lower than the first layer of monomers next to nanoparticle $\left(r=r_{c}\right.$ $+0.5 \sigma)$, followed by a second regime with a much higher slope. An almost constant population of the closest chain centers of mass in the first regime suggests that the total force between the closest chains and the particle is approximately zero. This is anticipated since long polymers can wrap around the particle and the forces on the average sum up to zero.

In the dilute regime, chains are depleted up to a range of around one $R_{g}$. At high polymer densities, short chains pack closely to the spherical filler in the dense regime and a peak in $g_{\text {col-cm }}(r)$ is found at $\left(r-r_{c}\right) / \sigma \approx 2$. In contrast, the particle may penetrate the longer chains and there is no packing of the chain centers of mass around the particle.
Additional information concerning the effect of the nanoparticle on the size and the orientation of the polymer chains can be extracted from the chain gyration tensor $\mathbf{S}$. For a chain of length $N$, the tensor is given by

$$
S_{i j}=\frac{1}{N} \sum_{m=1}^{N}\left(x_{i}^{m}-x_{i}^{\mathrm{cm}}\right)\left(x_{j}^{m}-x_{j}^{\mathrm{cm}}\right),
$$

where $\mathbf{x}^{m}$ and $\mathbf{x}^{\mathrm{cm}}$ are the position vector of monomer $m$ and the center of mass of the chain, respectively. The average size of a particular conformation is measured by the mean squared radius of gyration

$$
\left\langle R_{g}^{2}\right\rangle=\frac{1}{N}\left\langle\sum_{m=1}^{N}\left(\mathbf{x}^{m}-\mathbf{x}^{\mathrm{cm}}\right)^{2}\right\rangle .
$$

The explicit dimensions of the three chain semiaxes are determined by the eigenvalues of the chain gyration tensor. The sum of the three average eigenvalues $\left\langle\lambda_{i}\right\rangle,(i=1,2,3)$ gives the mean squared radius of gyration. The average dimension of the chain in each of its principal axes equals the square root of the respective eigenvalue. The three eigenvectors correspond to the orientation of each of the semiaxes defined by the particular polymer conformation.

In the polymer melt, polymers have the shape of flattened ellipsoids. ${ }^{50}$ In Ref. 8, the ratio of the three eigenvalues was reported to be 11.7:2.6:1, and a ratio of 12.0:2.7:1 is obtained for the random walk. In our simulations, the longest chains simulated $(N=120)$ have a ratio of $14.5: 3.0: 1$ at $\rho \sigma^{3}=0.025$ and 12.1:2.7:1 at $\rho \sigma^{3}=0.6$, which is in excellent agreement with the random walk. Clearly, at all densities the chains are flattened ellipsoids with dimensions and orientations that may be affected by the presence of the small particle.

The orientation of the chains relative to the surface of the colloid can be examined through the second Legendre polynomial $P_{2}$ between the vector connecting the center of the colloid and the center of mass of the chain, and the three eigenvectors of the radius of gyration tensor. Small chains tend to orient with their larger semiaxis parallel to the impenetrable surface and their smallest one perpendicular to it. In our simulations, chains are comparable or bigger than the colloid; such a preferential orientation is therefore only observed for the smallest chain length studied in this work. $P_{2}$ for the largest semiaxis of the molecules has a minimum of -0.10 for $N=16$ in the dilute regime which decreases to -0.20 in the melt regime. For longer polymers, $P_{2}$ was found to be close to zero at all densities, since chains can now wrap around the particle instead of orienting parallel to it.

Each eigenvalue of $\mathbf{S}$ was calculated as a function of the distance between the chain and particle centers of mass in order to observe any deformation in polymer size induced by the presence of the particle. Figure 9 presents the variation of the largest dimension of the chain $\left(\sqrt{\left\langle\lambda_{1}\right\rangle}\right)$ normalized by its value in the bulk $\left(\sqrt{\left\langle\lambda_{1, b}\right\rangle}\right)$.

As the polymer approaches the impenetrable surface of the colloid, three different regimes can be distinguished as shown schematically in Fig. 10. Far from the particle, the chain dimension is the bulk average value since the mol- 


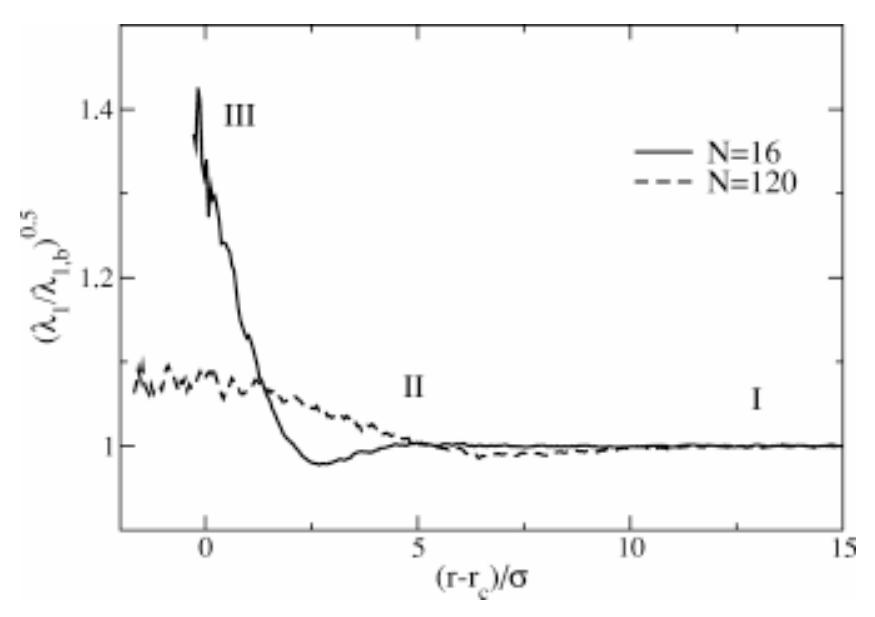

FIG. 9. Variation of the longest semiaxis of polymer ellipsoids relative to its bulk value, as a function of the colloid-chain center of mass distance for $N=16$ and $N=120$ at $\rho \sigma^{3}=0.1$.

ecules are not affected by the nanoparticle (region I). When the polymer coils approach at a distance comparable to their dimension they start to be distorted $\left(\sqrt{\left\langle\lambda_{1, b}\right\rangle}=2.17\right.$ and 7.12 for $N=16$ and $N=120$ at $\left.\rho \sigma^{3}=0.1\right)$. The polymer size along one of its semiaxis fluctuates around a mean value dictated by the corresponding eigenvalue therefore the colloid affects the molecules at even greater distances. In region II, a contraction of the chains take place at separations greater than their average size but lower than their maximum extension. As the center of mass of the chain moves even closer (region III), at shorter distances than the average size along a direction of the ellipsoids $\left(\sqrt{\left\langle\lambda_{1, b}\right\rangle}\right)$, then the polymer expands along the same direction. The chain size is distorted in each of the three axes when its center of mass is at distances similar to its dimension along the same axis. It is though by far the largest axis that mostly determines the spacial extension of the polymer molecule.

For small chains, a change in the orientation is also observed as the ellipsoids rotate parallel to the surface of the particle. As mentioned earlier, long chains do not rotate but instead wrap around the particle. Due to their greater size, chains with $N=120$ are distorted at longer distances than shorter chains but their maximum distortion is much smaller. As the filler becomes smaller relative to the polymers, it is expected that the induced deformation of the chains will decrease. It is also interesting to note that most of the chains increase their dimension mostly when their center of mass is out of the volume occupied by the particle. When the filler is

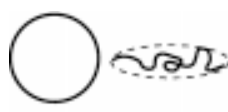

I

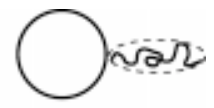

II

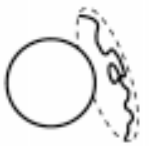

III

FIG. 10. Polymer size as a function of distance from the colloid. completely inside the chain, the polymer has expanded to an almost constant extent which is determined solely by the size of the filler. By increasing the density, the chain radius of gyration in the bulk decreases but the maximum extension of the chains relative to their size in the bulk remains almost the same. For the smallest chains of 16 beads long, the radius of gyration in the proximity of the particle can be up to 1.5 times larger than in the bulk. The longest polymers, consisting of 120 beads, are deformed only 1.1 times their average size in the absence of the nanoparticle.

\section{CONCLUSIONS}

The structure of a mixture of polymers and a single particle was investigated by off-lattice Monte Carlo simulations for chains with radius of gyration equal to or greater than the particle radius, and polymer densities from the dilute to the melt regime. The dependence of the range of the depletion interaction on chain length and polymer density were characterized by $\mathrm{MC}$ simulation and integral equation theory. At low densities, chain segments are strongly depleted in the vicinity of the filler. The monomer density profile is well described by mean-field equations and the depletion layer thickness extracted from simulations for different polymer lengths converges to a value of one bond length in dense polymer systems. Chain ends are found to be always preferentially located in the proximity of the impenetrable surface, an effect that becomes more pronounced with increasing chain lengths. Chain segments near the particle are found to prefer parallel alignment to the hard sphere surface for a range of approximately one segment length.

At high polymer densities, monomer packing results in an oscillatory particle-monomer pair distribution function. Chain ends are still found at higher than expected densities around the filler, while bonds form layers of parallel and perpendicular alignment relative to the surface of the particle.

When the macromolecules are long enough, the small particles are found to penetrate into the chains, as observed by the colloid-polymer center of mass pair distribution function. Packing effects for the centers of mass are observed only for the small chains due to their impenetrability, while for long chains the particle-chain center of mass correlation function displays an almost linear dependence on the center of mass separation.

The size and the orientation of the chains are examined by evaluating the radius of gyration tensor and finding the corresponding eigenvalues and eigenvectors. Polymer molecules are flattened ellipsoids at all densities studied. A contraction is observed when the filler is at a distance lower than the maximum but higher than the average size of the chain along one of its axis. At shorter distances than the mean size of the chain, an extension of the chains was found which is not greatly affected by the density. The deformation of the polymer molecules is much smaller for longer chains, while small chains also exhibit a preferential orientation parallel to the colloid.

Our simulations clearly show that chains deform in the vicinity of the filler, an effect that is expected to alter significantly the mechanical properties of the material at high poly- 
mer densities. We intend to extend our studies to the high density regime to polymeric solids and evaluate the possible changes in their elastic constants due to the presence of the filler. The effect of the polymer matrix on the interaction between two fillers will also be studied in order to extract information about the potential of mean force between two nanoparticles.

\section{ACKNOWLEDGMENTS}

This work is supported by a NIST grant from the National Science Foundation and by the Semiconductor Research Corporation.

${ }^{1}$ J. F. Joanny, L. Leibler, and P. G. D. Gennes, J. Polym. Sci., Polym. Phys. Ed. 17, 1073 (1979)

${ }^{2}$ A. Yethiraj, C. K. Hall, and R. Dickman, J. Colloid Interface Sci. 151, 102 (1992).

${ }^{3}$ R. Maasen, E. Eisenriegler, and A. Bringer, J. Chem. Phys. 115, 5292 (2001).

${ }^{4}$ M. Fuchs and K. S. Schweizer, J. Phys.: Condens. Matter 14, R239 (2002).

${ }^{5}$ A. A. Louis, P. G. Bolhuis, and E. J. Meijer, J. Chem. Phys. 116, 10547 (2002).

${ }^{6}$ M. S. Ozmusul and R. C. Picu, Polymer 43, 4657 (2002).

${ }^{7}$ J. Klos and T. Pakula, J. Chem. Phys. 118, 1507 (2003).

${ }^{8}$ R. C. Picu and M. S. Ozmusul, J. Chem. Phys. 118, 11239 (2003).

${ }^{9}$ A. Chatterjee and K. S. Schweizer, J. Chem. Phys. 109, 10464 (1998).

${ }^{10}$ S. Ramakrishnan, M. Fuchs, K. S. Schweizer, and C. F. Zukoski, J. Chem. Phys. 116, 2201 (2002).

${ }^{11}$ E. J. Meijer and D. Frenkel, J. Chem. Phys. 100, 6873 (1994).

${ }^{12}$ M. Dijkstra, J. M. Brader, and R. Evans, J. Phys.: Condens. Matter 11, 10079 (1999).

${ }^{13}$ V. J. Anderson and H. N. W. Lekkerkerker, Nature (London) 416, 811 (2002).

${ }^{14}$ W. C. K. Poon, J. Phys.: Condens. Matter 14, R859 (2002).

${ }^{15}$ S. A. Shah, Y. L. Chen, K. S. Schweizer, and C. F. Zukoski, J. Chem. Phys. 118, 3350 (2003).

${ }^{16}$ P. G. Bolhuis, A. A. Louis, and J.-P. Hansen, Phys. Rev. Lett. 89, 128302 (2002).

${ }^{17}$ P. G. Bolhuis, E. J. Meijer, and A. A. Louis, Phys. Rev. Lett. 90, 068304 (2003).

${ }^{18}$ O. Guzmán and J. J. de Pablo, J. Chem. Phys. 118, 2392 (2003).

${ }^{19}$ P. G. Bolhuis and A. A. Louis, Macromolecules 35, 1860 (2002).

${ }^{20}$ A. A. Louis, P. G. Bolhuis, and E. J. Meijer, J. Chem. Phys. 117, 1893 (2002).
${ }^{21}$ M. Vacatello, Macromolecules 34, 1946 (2001).

${ }^{22}$ M. Vacatello, Macromolecules 35, 8191 (2002).

${ }^{23}$ M. Vacatello, Macromolecules 36, 3411 (2003).

${ }^{24}$ F. A. Escobedo and J. J. de Pablo, J. Chem. Phys. 102, 2636 (1995).

${ }^{25}$ Z. Chen and F. A. Escobedo, J. Chem. Phys. 113, 11382 (2000).

${ }^{26}$ A. Uhlherr, V. G. Mavrantzas, M. Doxastakis, and D. N. Theodorou, Macromolecules 34, 8554 (2001).

${ }^{27}$ P. V. K. Pant and D. N. Theodorou, Macromolecules 28, 7224 (1995).

${ }^{28}$ V. G. Mavrantzas, T. D. Boone, E. Zervopoulou, and D. N. Theodorou, Macromolecules 32, 5072 (1999).

${ }^{29}$ A. Uhlherr, M. Doxastakis, V. G. Mavrantzas, D. N. Theodorou, S. J. Leak, N. E. Adam, and P. E. Nyberg, Europhys. Lett. 57, 506 (2002).

${ }^{30}$ A. Uhlherr, S. J. Leak, N. E. Adam, P. E. Nyberg, M. Doxastakis, V. G. Mavrantzas, and D. N. Theodorou, Comput. Phys. Commun. 144, 1 (2002).

${ }^{31}$ M. Doxastakis, V. G. Mavrantzas, and D. N. Theodorou, J. Chem. Phys. 115, 11339 (2001).

${ }^{32}$ M. Doxastakis, V. G. Mavrantzas, and D. N. Theodorou, J. Chem. Phys. 115, 11352 (2001)

${ }^{33}$ R. Faller, F. Müller-Plathe, M. Doxastakis, and D. N. Theodorou, Macromolecules 34, 1436 (2001).

${ }^{34}$ P. Gestoso, E. Nicol, M. Doxastakis, and D. N. Theodorou, Macromolecules 36, 6925 (2003).

${ }^{35}$ N. C. Karayiannis, V. G. Mavrantzas, and D. N. Theodorou, Phys. Rev. Lett. 88, 105503 (2002).

${ }^{36}$ B. J. Banaszak and J. J. de Pablo, J. Chem. Phys. 119, 2456 (2003).

${ }^{37}$ J. P. Hansen and I. R. McDonald, Theory of Simple Liquids (Academic, London, 1986).

${ }^{38}$ S. A. Shah, Y.-L. Chen, S. Ramakrishnan, K. S. Schweizer, and C. F. Zukoski, J. Phys.: Condens. Matter 15, 4751 (2003).

${ }^{39}$ S. A. Shah, S. Ramakrishnan, Y.-L. Chen, K. S. Schweizer, and C. F. Zukoski, Langmuir 19, 5128 (2003).

${ }^{40}$ K. Schweizer and J. Curro, Adv. Polym. Sci. 116, 319 (1994).

${ }^{41}$ K. Schweizer and J. Curro, Adv. Chem. Phys. 98, 1 (1997).

${ }^{42}$ K. Honnell, J. Curro, and K. Schweizer, Macromolecules 23, 496 (1990).

${ }^{43}$ J. Percus and G. Yevick, Phys. Rev. 110, 1 (1958).

${ }^{44}$ P. deGennes, Scaling Concepts in Polymer Physics (Cornell University Press, Ithaca, 1979).

${ }^{45}$ M. Fuchs and K. S. Schweizer, Europhys. Lett. 51, 621 (2000).

${ }^{46}$ M. Fuchs and K. S. Schweizer, Phys. Rev. E 64, 021514 (2001).

${ }^{47}$ D. G. A. L. Aarts, R. Tuinier, and H. N. W. Lekkerkerker, J. Phys.: Condens. Matter 14, 7551 (2002).

${ }^{48}$ G. J. Fleer, A. M. Skvortsov, and R. Tuinier, Macromolecules 36, 7857 (2003).

${ }^{49}$ S. K. Kumar, M. Vacatello, and D. Y. Yoon, J. Chem. Phys. 89, 5206 (1988)

${ }^{50}$ D. N. Theodorou and U. W. Suter, Macromolecules 18, 1206 (1984). 